HeatMeUp: a 3DUI Serious Game to Explore Collaborative Wayfinding Peer-reviewed author version

NOTELAERS, Sofie; DE WEYER, Tom; GOORTS, Patrik; MAESEN, Steven;

VANACKEN, Lode; CONINX, Karin \& BEKAERT, Philippe (2012) HeatMeUp: a 3DUI

Serious Game to Explore Collaborative Wayfinding. In: Proceedings of the IEEE

Symposium on 3D User Interfaces 2012, p. 177-178.

Handle: http://hdl.handle.net/1942/13737 


\title{
HeatMeUp: a 3DUI Serious Game to Explore Collaborative Wayfinding
}

\author{
Sofie Notelaers* Tom De Weyer ${ }^{\dagger} \quad$ Patrik Goorts ${ }^{\ddagger} \quad$ Steven Maesen ${ }^{\S} \quad$ Lode Vanacken ${ }^{\mathbb{q}}$ \\ Karin Coninx\| Philippe Bekaert** \\ Hasselt University - tUL - IBBT \\ Expertise Centre for Digital Media
}

\begin{abstract}
Wayfinding inside a virtual environment is a cognitive process during navigation. Normally the user inside the virtual environment has to rely on himself and different cues such as waypoints to improve his knowledge with regard to his surroundings. In this paper we will present our solution for the 3DUI Contest 2012: HeatMeUP, a 3DUI serious game to explore collaborative alternatives, in which a partner is responsible for providing wayfinding cues. The game is set in a multi-storey building where several fires and gas leaks occur and a firefighter has to overcome several challenges, guided by a fire chief.
\end{abstract}

\section{INTRODUCTION}

3DUI serious games are an interesting opportunity to explore different types of interaction in a virtual setting. More specific, games which have a focus towards simulation purposes provide the ability to easily test and replay different scenarios. These properties allow people playing the game to potentially provide valuable feedback which can be used in the similar real life situation. For the 3DUI Contest 2012, we decided to start from a scenario of a fire inside a complex multi-storey building to make such a game.

Our 3DUI serious game, HeatMeUp, is set in a multi-storey building, which a firefighter needs to enter to ultimately save a person. In order to be able to complete his task successfully he will have to overcome several challenges such as fires, gas leaks, structural collapses, closed doors and toxic smoke. In order to be able to do this, several instruments need to be found inside the building, such as fire extinguishers, valves controlling the gas lines, a passkey, a gas mask, etc. Even though several of these objects will have similar positions across several floors, it is not straightforward to find your way alone across floors without any prior knowledge of the building. From the control room at a different location, the fire chief will have to help the firefighter without traditional communication channels, as we assume them to be unavailable in our specific situation.

\section{HeatMeUp: a 3DUI Serious Game}

The goal of the game is to save a person inside a multi-storey building while overcoming typical challenges induced in fire situations. In order to be able to overcome these challenges more easily, the fire chief is helping out by providing wayfinding hints to the firefighter inside the building. Hence two set-ups are created which

\footnotetext{
*e-mail: sofie.notelaers@uhasselt.be

†e-mail: tom.deweyer@uhasselt.be

†e-mail: patrik.goorts@uhasselt.be

§e-mail: steven.maesen@uhasselt.be

$\mathbb{I}_{\text {e-mail: lode.vanacken@uhasselt.be }}$

"e-mail: karin.coninx@uhasselt.be

**e-mail: philippe.bekaert@uhasselt.be
}

respectively provide an interface for the firefighter inside the building and the fire chief inside the control room. An overview of both set-ups can be found in figure $1(a-b)$.

\subsection{The Firefighter Set-up}

The set-up for the firefighter uses a 21-inch widescreen LCD to display the virtual world, while a Logitech gamepad is used to be able to navigate and perform actions inside the world. In order to try and enhance the feeling of embodiment inside the world we augmented this set-up with a system to simulate heat. The system activates when the firefighter would come in close contact with a hot situation (i.e. a fire or a gas leak), it would make sure that the heat could be felt. In order to dissipate heat, 150W lamps were used, which are controlled with a DMX control board [2].

The firefighter's set-up is designed to create a feeling of discomfort near fires. Other existing simulators are typically set in existing VR hardware such as CAVE [1] or a desktop PC [4, 3] and do not really try to immerse the firefighter using different modalities.

The firefighter can travel through the environment and pick up items. These items are highlighted when standing close to them and can be picked up by pressing the action button. Items which allow an action are highlighted similarly and mapped to the same button on the game controller. When the firefighter picks up a general item, he will always carry it along and will be able to use it during the entire game, such as, for example, a passkey to open all doors as well as a gas mask to be able to travel through toxic smoke. The only exception is a fire extinguisher, which depletes after having extinguished two fires.

Wayfinding metaphors are the remaining important aspect inside the navigation capabilities provided to the firefighter. In our solution the firefighter can only undergo wayfinding metaphors provided by the control room by means of the lighting system. His current location and viewpoint is provided to the control room.

\subsection{The Control Room Set-up}

The control room set-up consists of three areas, of which two provide only information about the environment and the remaining one also provides interaction capabilities. The interaction area is a 2D Philips 6TP 42-inch multi-touch screen which is used as a flat surface to show the floor plans of the building. On this screen multitouch interaction will provide wayfinding possibilities as well as annotation possibilities to annotate a possible updated situation on the floor plan (a gas leak, a collapse, ...). The other two areas are both views into the virtual world. The left view shows the current situation of the firefighter. On the right, a view is given on a remotely controlled vehicle (RCV) which can be controlled from the multi-touch screen. This vehicle will provide functionalities to detect hazardous situations as well as explore areas before the firefighter can easily reach or enter them.

In figure 1(c) the multi-touch interaction area is visualized. The blueprint of each floor can be dragged upon the interaction area. On such a floor plan, the current position of the firefighter and the RCV are shown. The information available beforehand is automatically visualized on the floor plans; these include the positions of the fires, acquired from fire alarms; and the position of objects, such as 

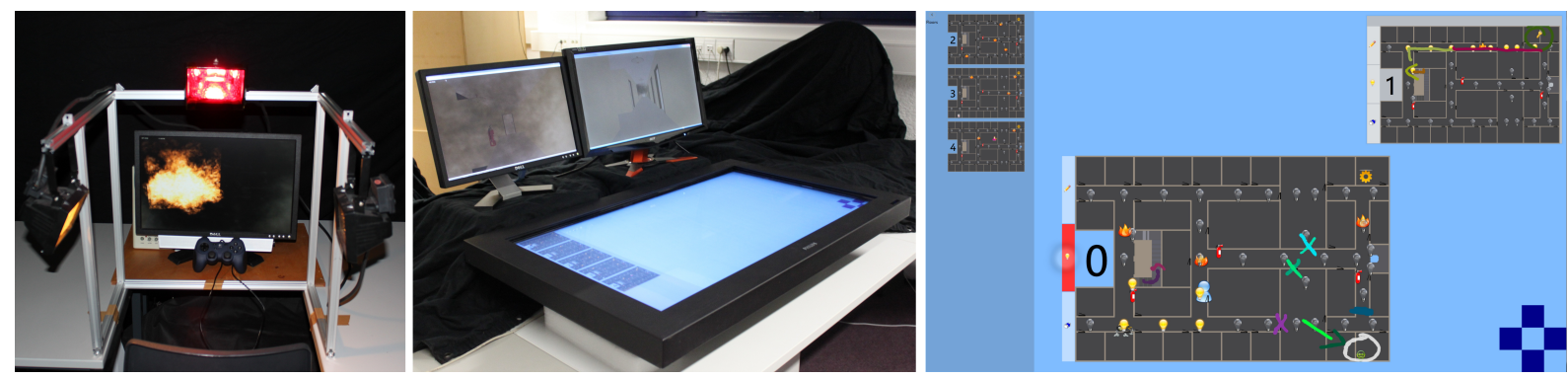

Figure 1: (a) Overview of the firefighter set-up (b) Overview of the fire chief set-up (c) The multi-touch interaction area

lights, doors, fire extinguishers, etc. Each floor plan has menu items on the left-hand side. These have to be continuously touched, just like holding a piece of paper when writing on it, to define the active action. Three actions are currently available: adding and removing annotations, and turning lights on and off. The annotations are not visible for the firefighter, while the lights are controlled in the virtual environment to provide wayfinding clues. Finally, in the lower right-hand corner of the multi-touch screen, a gamepad like interface is used to steer the RCV throughout the virtual environment.

\section{Informal Pilot Study}

To evaluate our solution we performed an informal pilot study. We used the set-ups discussed in the previous section. 4 users (one female and three males) served as participants in this experiment. Participants were selected among co-workers and students, with ages between 24 and 30 . The participants were paired randomly and only took part once, hence they would only either be a firefighter or the fire chief inside the control room. None of the participants had any experience with regard to their role nor had they extensive 3DUI experience.

A 5 -storey building ( 34 by 22 meter) with several collapses, fires, a toxic floor (which needed a gas mask to allow entering this floor), locked doors and a first aid kit was designed. Even though each floor is based on an initial design, the combination with all obstacles would provide a far from straightforward path throughout the environment, hence a maze like feeling was created. The participants were informed that the goal was to reach the fifth floor with the first aid kit, which resided somewhere in the building, in order to rescue the person on that particular floor. Note that the design was made such that it was necessary to go up and down floors to gather items required for entering restricted areas (i.e. closed doors and toxic smoke). During their rescue operation they would have to help each other out to reach the goal by means of using the interaction techniques provided by our solution. They were given separate instructions depending on the role they played and had some time to practice until they felt comfortable in using the solution. Their performance was observed while time and distance were logged. Finally, the experiment concluded with an semi-structured interview.

\section{Results}

Both groups had similar performance results. The first group took 1360 seconds and traveled 1374 meters to complete the task. The second group needed 1291 seconds and traveled 1320 meters. Clearly the design of the building and the task was pretty complex and collaboration between both roles was necessary to be able to complete the task. Furthermore the travel distance indicated that users had to move around a lot and search their way throughout the building. Worth mentioning is that completing the game in an almost ideal path would take about 490 meters and 300 seconds.

During the study we could observe several interesting findings. Fire chiefs initially had difficulties planning the path while simultaneously observing the firefighter, especially if they also wanted to make annotations of obstructions. Their primary concern was controlling the lights so that they regularly missed blockages which could be observed in the view of the firefighter. Near the end their multi-tasking performance did improve, indicating that a rather steep learning curve is present. It is hard to judge whether this is due to lack in experience of multi-tasking or using the control room set-up. Moreover we observed that one of the firefighters used the heat feedback to not come too near to the fires and extinguish them from a safe distance. The other firefighter suggested that even more feedback would be pleasant. Although not instructed during the training period, the chiefs flicked lights to draw the attention of the firefighters. This showed to be convenient for firefighters which had lost track of their direction/orientation inside a hall-way.

The interview revealed that the participants were overall pleased with the solution. One of the firefighters was surprised that so much information could be transferred only using the lights in the scene. Both found that heat feedback really gave an immersive feeling and that it made the game more realistic.

Besides judging the capabilities of turning lights on or off to provide wayfinding cues, both firefighters suggested more detailed feedback for entering rooms would be valuable. Sometimes it was not clear if they had to enter one of the rooms, or proceed further down the hall. They instructed that the origin of this issues was very likely due to the fact that the fire chief was not fast enough in lighting up the path in the hallway and more importantly had no means to indicate that they should wait for further instructions. This fact was further enforced by one of the comments of a fire chief, which suggested that some kind of signal was needed to make clear that the firefighter should be stationary for a while.

\section{Discussion}

From our informal pilot study we can convey several interesting findings. First of all, lighting up a path was found to be very convenient and usable to perform wayfinding cues. Secondly, the heat dissipation was judged to add extra realism to the presented solution. Thirdly, making annotations while providing wayfindings cues as well as steering the RCV was found to be tedious. Finally, including an extra information channel towards the firefighter could provide other relevant information such as 'wait for a while'. This could for instance be conveyed using lightweight tactile feedback built into the firefighting suit.

\section{REFERENCES}

[1] P. Backlund, H. Engstrm, M. Gustavsson, M. Johannesson, M. Lebram, and E. Sjörs. Sidh: A game-based architecture for a training simulator. International Journal of Computer Games Technology, 2009.

[2] A. Bennette. Recommended Practice for DMX512. PLASA, 2006.

[3] J. Dugdale, B. Pavard, N. Pallamin, M. el Jed, and C. Maugan. Emergency fire incident training in a virtual world. In Proceedings of ISCRAM'04), 2004.

[4] T. St Julien and C. Shaw. Firefighter command training virtual environment. In Proceedings DIC'03, pages 30-33. ACM, 2003. 